\title{
Análise probabilística da vida útil de lajes de concreto armado sujeitas à corrosão por carbonatação via simulação de Monte Carlo
}

\author{
Probabilistic analysis of the useful life of \\ reinforced concrete slabs subjected to \\ carbonation corrosion by Monte Carlo simulation
}

Emerson Felipe Felix ${ }^{1}$, Rogério Carrazedo ${ }^{1}$

\footnotetext{
${ }^{1}$ Departamento de Engenharia de Estruturas, Escola de Engenharia de São Carlos, Universidade de São Paulo, Av. Trabalhador Sancarlense, 400, Centro, CEP 13566-590, São Carlos, SP, Brasil. e-mail: emerson.felipe.felix@ gmail.com, rogcarrazedo@sc.usp.br
}

\section{RESUMO}

O presente trabalho apresenta uma análise mecânico-probabilística de lajes de concreto armado sujeitas à corrosão uniforme, devido à ação do $\mathrm{CO}_{2}$ no concreto, por meio de uma metodologia alternativa que considera formulações analíticas referentes ao comportamento mecânico do concreto acopladas a modelos obtidos via Inteligência Artificial e à técnica de simulação de Monte Carlo. Os períodos de iniciação e propagação da corrosão são determinados por meio do cálculo de probabilidades de ocorrência dos estados limites de despassivação e ruptura à flexão, respectivamente. Os resultados encontrados apontam que, a depender do controle tecnológico do concreto, do cobrimento e do ambiente de exposição, a vida útil pode ser drasticamente reduzida, apresentando em muitos casos confiabilidade estrutural e durabilidade inferior às requisitadas em projeto. A fim de garantir menor probabilidade de falha devido à corrosão por carbonatação, recomenda-se empregar cimentos Portland do tipo CP V, CP II F, CP II E e CP II Z, para a produção de estruturas inseridas em ambientes com alta concentração de $\mathrm{CO}_{2}$. Além disso, os resultados mostram que abordagens puramente determinísticas não são capazes de avaliar adequadamente a vida útil de estruturas de concreto sujeitas ao processo de corrosão, sendo necessário analisar o problema considerando a aleatoriedade dos diferentes parâmetros associados ao processo de deterioração, a fim de representar com fidelidade o processo de corrosão e seus efeitos deletérios.

Palavras-chave: lajes de concreto armado, degradação do concreto, corrosão, simulação de Monte Carlo.

\begin{abstract}
In this work we propose a coupled mechanical-probabilistic analysis of the useful life of reinforced concrete slabs under uniform corrosion due to $\mathrm{CO}_{2}$ diffusion. The alternative approach considers analytical formulations for the mechanical behavior of concrete coupled to a model based on Artificial Intelligence and the Monte Carlo simulation. The initiation and propagation periods are determined by the probability of occurrence of the limit states of depassivation and flexural strength, respectively. The results show that depending on the quality control of concrete, concrete cover and exposure environment, the useful life can be drastically reduced. In many cases the structural reliability and durability are lower than the specified in design. Thus, it is recommended to use Brazilian Portland cements type CP V, CP II F, CP II E and CP II Z (or equivalents) for the production of concrete structures in environments with high $\mathrm{CO}_{2}$ concentration. Furthermore, we show that that purely deterministic approaches are not able to adequately assess the useful life of concrete structures under corrosion. One must consider the randomly distribution of the parameters associates to the deterioration process to accurately represent the corrosion process and its consequences.
\end{abstract}

Keywords: reinforced concrete slab, concrete deterioration, corrosion, Monte Carlo simulation. 


\section{INTRODUÇÃO}

O crescimento econômico e o desenvolvimento sustentável da sociedade são fatores dependentes da infraestrutura civil, a qual requer uma adequada gestão de sua durabilidade a fim de proporcionar estruturas confiáveis e seguras [1]. No entanto, os sistemas e instalações de infraestrutura são inerentemente vulneráveis e estão expostos a diferentes mecanismos deteriorativos que fazem com que a sua vida útil seja reduzida, a exemplo de deteriorações devido a ataques químicos (corrosão, ataque por sulfatos e reação álcali-agregado), mecanismos físicos (expansão térmica ou retração), mecanismos biológicos (desenvolvimento de fungos e bactérias) e mecanismos mecânicos (fadiga e carregamento excessivo) [2, 3]. Neste aspecto, percebe-se a importância do conceito de vida útil, que serve como base para uma abordagem holística de projeto.

A corrosão é uma das principais causas de redução da vida útil de estruturas de concreto armado [3]. De acordo com a organização mundial da corrosão "World Corrosion Organization", os custos envolvidos com esta manifestação patológica alcançam valores superiores a 3\% do Produto Interno Bruto (PIB) em diversos países, e a incidência deste mecanismo deteriorativo em estruturas de concreto armado se destaca dentre os demais, sendo observados índices de ocorrência de até $48 \%$ na África do Sul, $25 \%$ no Reino Unido, $36 \%$ na Índia e 31\% nos Estados Unidos [4]. No Brasil, por exemplo, a corrosão possuí um índice de ocorrência que varia de 14 a $64 \%$, a depender da região de análise [5, 6].

A corrosão sofrida pelas armaduras decorrente do processo de oxidação é provocada pela reação gásmetal que dá origem a uma película de óxido que envolve o elemento metálico. Este tipo de corrosão possui um processo de evolução lento quando em ambiente de exposição à temperatura ambiente $\left( \pm 25^{\circ} \mathrm{C}\right)$, salvo em condições de exposição de agressividade severa ou na presença de gases extremamente agressivos presentes na atmosfera, como o $\mathrm{CO}_{2}$ e íons cloreto [7, 8].

A corrosão por carbonatação, devido à difusão de $\mathrm{CO}_{2}$ na matriz do concreto, se desenvolve uniformemente na seção longitudinal da armadura. O processo corrosivo ocorre sob velocidade controlada pela taxa de corrosão, sendo dependente de diversos fatores, sejam eles ambientais ou relativos a parâmetros referentes às propriedades do material [9].

Os principais fatores ambientais são a concentração de $\mathrm{CO}_{2}$ presente na atmosfera, a incidência de chuvas, a umidade relativa do ar e a temperatura [6]. Quanto aos fatores internos, citam-se a relação água/cimento empregada na produção do concreto, a sua resistência à compressão, a espessura de cobrimento das armaduras e a composição do concreto [4].

ANDRADE [6] descreve três formas de manifestação dos efeitos da corrosão sobre o material compósito, sendo eles: a redução da capacidade mecânica, a perda de aderência entre aço e concreto e a fissuração e/ou destacamento do concreto de cobrimento.

De acordo com HELENE [8], a corrosão desencadeada pela carbonatação, que se configura como corrosão uniforme, é um processo usualmente visto em estruturas situadas em ambiente urbano, podendo ainda ter forte incidência em regiões litorâneas, dependendo do nível de concentração de íons cloreto na atmosfera. No que condiz o processo corrosivo iniciado pela carbonatação, este é classificado por duas fases, a iniciação e a propagação, sendo a transição caracterizada pela despassivação do aço, conforme mostra a Figura 1.

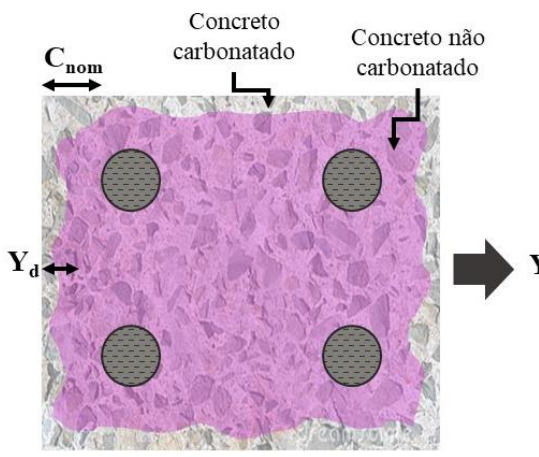

(a) Antes da corrosão $\mathbf{Y}_{\mathbf{d}}<\mathbf{C}_{\text {nom }}$

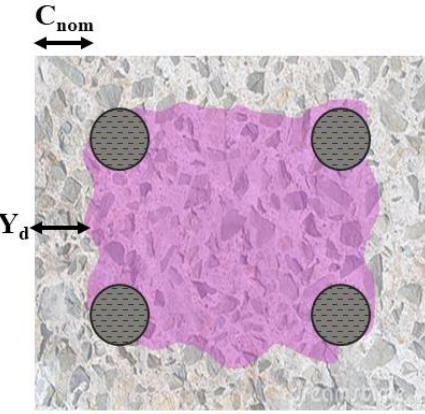

(b) Despassivação - inicio da corrosão $\mathbf{Y}_{\mathrm{d}}=\mathbf{C}_{\text {nom }}$

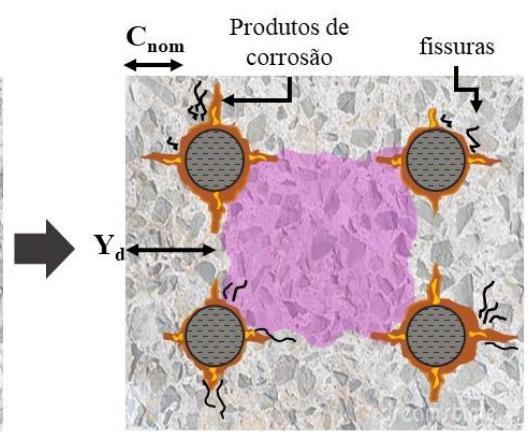

(c) Propagação da corrosão $\mathbf{Y}_{\mathrm{d}}>\mathbf{C}_{\mathrm{nom}}$

Figura 1: Avanço da corrosão por carbonatação em uma estrutura de concreto armado.

A iniciação da corrosão é definida pelo instante em que ocorre a despassivação da armadura. No caso da corrosão por carbonatação, assume-se que isso ocorre no instante de tempo em que o $\mathrm{CO}_{2}$ alcança a superfície do aço. O processo de carbonatação pode ser caracterizado, de forma simplificada, pela iteração química 
entre o $\mathrm{CO}_{2}$ e o hidróxidos de cálcio $\left(\mathrm{Ca}(\mathrm{OH})_{2}\right)$ contido na pasta de cimento, tendo como produto da reação o carbonato de cálcio $\left(\mathrm{CaCO}_{3}\right)$ [10-13]. A carbonatação, além de causar a despassivação do aço, pode reduzir a porosidade e permeabilidade do concreto $[10,11]$.

Após a despassivação do aço, dá-se início o período de propagação, que é caracterizado pela taxa de corrosão e a qualidade do concreto de cobrimento, principal elemento que controla as tensões internas que surgem na região de contato aço/concreto devido ao surgimento dos produtos de corrosão (óxido férrico) [14, 15]. A expansão ocasionada pela formação do óxido férrico causa a fissuração do concreto de cobrimento, devido ao campo de tensão que é induzido na região de contato entre os dois materiais. Em função do nível da tensão de tração gerada pelo desenvolvimento dos produtos de corrosão, macrofissuras se formam, facilitando o ingresso dos agentes agressivos presentes na atmosfera, ocasionando uma ascensão no nível de degradação do material e, consequentemente, na redução de sua vida útil [14]. Somado à fissuração e danificação do concreto, DU et al. [16] relatam o efeito combinado da corrosão e do carregamento externo, que provoca uma redução da resistência residual da armadura, a qual é desproporcional à taxa de perda de seção útil de aço.

No que condiz a modelagem do fenômeno da corrosão por carbonatação, a maioria dos trabalhos existentes na literatura têm como escopo o estudo do período de iniciação da corrosão, principalmente no desenvolvimento de modelos representativos da difusão do $\mathrm{CO}_{2}$ no concreto, ou do tempo necessário para ocorrer a despassivação do aço imerso no concreto $[12,13,17-20]$. Uma justificativa para o desenvolvimento de modelos representativos da fase de iniciação se deve ao fato de que uma correta estimativa do tempo de despassivação do aço propicia uma melhor avaliação do grau de deterioração das estruturas de concreto armado e da vida útil residual do material compósito. Ademais, a utilização destes modelos, durante fase de projeto, possibilita estimar a vida útil de projeto de uma estrutura de concreto e tomar medidas que possibilitem mitigar ou minimizar a corrosão de suas armaduras, já que o projetista poderá alterar a composição do concreto por meio da incorporação de adições ou adotar aditivos inibidores [21].

Assim, mesmo sob a existência de diversos modelos representativos da corrosão [3, 4, 12-20], as estimativas dos períodos de despassivação do aço ainda apresentam erros associados à falta de generalidade dos modelos no que diz respeito às condições de exposição e/ou aspectos climáticos do ambiente que circunda as estruturas de concreto [22]. Ademais, LIBERATI et al. [22] e RAMEZANIANPOUR et al. [23] relatam a falta de representação das incertezas presentes em parâmetros associados às condições climáticas do ambiente, sendo necessário, portanto, acrescentar variáveis aleatórias que descrevam a variabilidade destes parâmetros.

Para contornar a falta de representação da variabilidade dos parâmetros dos modelos determinísticos, RAMEZANIANPOUR et al. [23] relata que análises via teoria da confiabilidade podem ser conduzidas com estes modelos, a fim de estimar a probabilidade de ocorrência da corrosão ou de despassivação do aço com maior fidelidade, inserindo nos resultados fatores relacionados a aleatoriedade das variáveis que influenciam no problema. Os autores relatam que abordagens puramente determinísticas podem apresentar resultados com baixo índice de confiabilidade quando o ambiente circundante da estrutura apresenta grandes variações em suas condições climáticas.

Atualmente existem diversos métodos para avaliar a performance e confiabilidade de estruturas de concreto frente à variação de suas propriedades mecânicas e de durabilidade [1,24-26]. A técnica de simulação Monte Carlo constitui-se como um método poderoso para essa análise, já que considera simultaneamente os diferentes parâmetros aleatórios envolvidos no problema [27, 28]. Assim, a associação de formulações matemáticas representativas das leis de difusão a algoritmos de confiabilidade apresenta uma metodologia eficiente para a modelagem e estimativa da vida útil de estruturas de concreto sujeitas à corrosão, com resultados mais consistentes, abrangentes e confiáveis que as estimativas determinísticas [1, 22-29].

Assim, considerando o crescente emprego da teoria da confiabilidade na análise probabilística da falha de componentes estruturais devido à ação de diferentes mecanismos de deterioração, em especial a corrosão das armaduras, este trabalho apresenta uma metodologia alternativa para a análise da vida útil de estruturas de concreto armado submetidas à corrosão por carbonatação. Como inovação, a metodologia proposta para o cálculo da probabilidade de falha mecânica, de estruturas de concreto armado sujeitas à corrosão, acopla um modelo representativo da difusão do $\mathrm{CO}_{2}$ no concreto, obtido via Inteligência Artificial, à técnica de simulação de Monte Carlo. A probabilidade de falha é determinada considerando os princípios da teoria da confiabilidade, associada a modelos referentes aos processos de carbonatação, despassivação do aço, perda de seção útil da armadura e à capacidade mecânica de elementos de concreto armado. Para demonstrar a eficiência do método, é apresentado um estudo de caso no qual são analisadas lajes de concreto armado localizadas em uma região urbana da cidade de São Paulo, considerando concretos produzidos com diferentes tipos de ci- 
mento Portland (CP II-E, CP II-F, CP II-Z, CP III, CP IV e CP V) e distintas condições de exposição (ambiente interno ou externo e estruturas protegidas ou desprotegidas da chuva).

\section{MODELAGEM MECÂNICO-PROBABILISTÍCA}

Com o objetivo de descrever detalhadamente as etapas da metodologia proposta para a modelagem via simulação de Monte Carlo, são descritas na sequência os modelos utilizados na representação do processo de carbonatação do concreto, despassivação das armaduras, perda de área útil de aço e da falha à flexão de lajes de concreto armado. São apresentadas ainda, o acoplamento destas formulações à metodologia de análise probabilística e a definição das funções de distribuição das variáveis aleatórias do estudo de caso.

\subsection{Modelo implementado para a perda de seção de aço}

Como objetiva-se analisar lajes de concreto susceptíveis à corrosão uniforme, o diâmetro da armadura, $\phi$, sofre uma redução ao longo do tempo, sendo necessário determiná-la a cada instante de análise. Dessa forma, foram utilizadas formulações que fornecessem o diâmetro integro de aço [30] e da taxa de corrosão [31] ao longo do tempo, dadas respectivamente nas Equações 1 e 2.

$$
\begin{aligned}
& \phi\left(t_{p}\right)=\phi_{0}-\left(2 \cdot 0,0116 \cdot \eta \cdot t_{p}\right) \\
& \eta=\left(\frac{37,8 \cdot(1-a / c)^{-1,64}}{c o b}\right) \cdot 0,85 \cdot\left(t_{p}\right)^{-0,29}
\end{aligned}
$$

em que $\phi\left(t_{p}\right)$ é o diâmetro integro de aço (em $\mathrm{mm}$ ) no tempo $t_{p}$ (em anos) após iniciar o período de progressão da corrosão, e tem valor máximo calculado pela Equação $3, \phi_{0}$ é o diâmetro inicial da barra $(\mathrm{mm}), \eta$ representa a taxa de corrosão da armadura $\left(\mu \mathrm{A} / \mathrm{cm}^{2}\right), a / c$ é a relação água/cimento do concreto e $c o b$ é a espessura de cobrimento do concreto $(\mathrm{mm})$.

$$
t_{p} \leq\left\{\begin{array}{c}
75-t_{\text {ini }} \quad \text { se } t_{\text {ini }}<75 \\
0 \text { se } t_{\text {ini }} \geq 75
\end{array}\right.
$$

em que $t_{i n i}$ é o instante de tempo em que ocorre a despassivação do aço. Destaca-se que neste trabalho, o tempo de análise máximo é de 75 anos, referente ao período que compreende o tempo mínimo (50 anos) e superior (75 anos) de vida útil de projeto prescrito na NBR 15575-1 [32]. Caso a despassivação ocorra após este período, o tempo de progressão é definido como nulo, conforme mostra a Equação 3.

Para a determinação do instante em que ocorre o início da degradação das armaduras pela corrosão e, consequentemente, a sua despassivação, $t_{i n i}$, acoplou-se à Equação 3 um modelo numérico de predição da profundidade de carbonatação do concreto proposto em FELIX et al. [33]. O modelo foi desenvolvido via Redes Neurais Artificiais com redes do tipo multilayer perceptron, sendo apresentado na sequência.

\subsection{Modelo da carbonatação do concreto}

O modelo de predição da profundidade de carbonatação do concreto foi obtido por meio do treinamento com Redes Neurais Artificiais (RNA) do tipo MultiLayer Perceptron (MLP), utilizando o algoritmo de treinamento backpropagation. A descrição completa da metodologia e dos parâmetros utilizados na modelagem encontra-se em FELIX et al. [33].
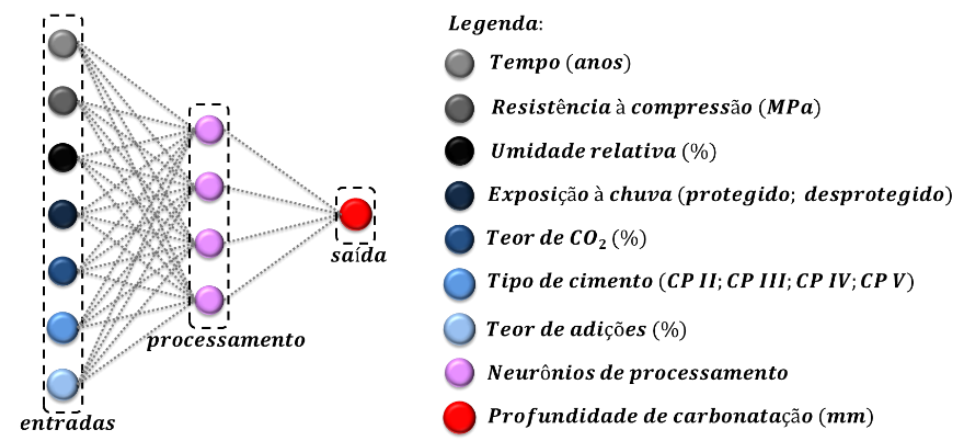

Figura 2: Topologia da rede neural artificial do modelo predição da profundidade de carbonatação. 


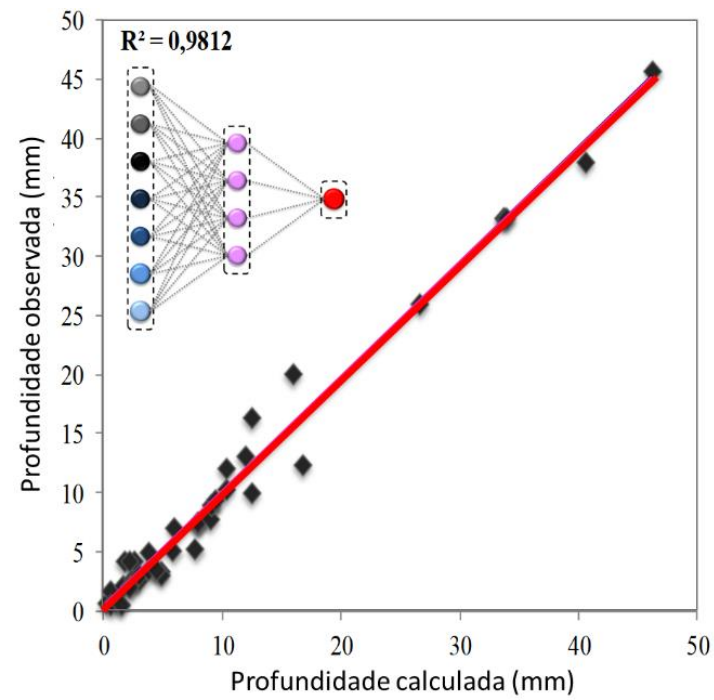

Figura 3: Performance do modelo utilizado para a carbonatação do concreto.

Após treinar, validar e testar 91 redes, FELIX et al. [33] selecionaram a que melhor representava o comportamento da frente de carbonatação do concreto. O modelo escolhido possui a topologia apresentada na Figura 2, contendo três camadas de neurônios. Na primeira estão dispostos sete neurônios que fazem referência às variáveis de entrada, na segunda camada existem quatro neurônios responsáveis pelo processamento das informações e, na terceira camada, um neurônio referente à saída da rede: a profundidade de carbonatação.

O coeficiente de determinação de 0,98 , obtido com os resultados da análise de performance do modelo, demonstra o seu grau de aplicabilidade frente à estimativa da carbonatação de concretos situados no ambiente urbano brasileiro, validando a sua utilização no presente trabalho.

\subsection{Propriedades estatísticas das variáveis}

Para a análise probabilística via simulação de Monte Carlo, faz-se necessário descrever as variáveis aleatórias do problema de estudo e suas funções de distribuição. Neste trabalho, são utilizadas como variáveis aleatórias: a umidade relativa do ar, o teor de $\mathrm{CO}_{2}$ na atmosfera, a resistência à compressão do concreto e o cobrimento nominal das armaduras.

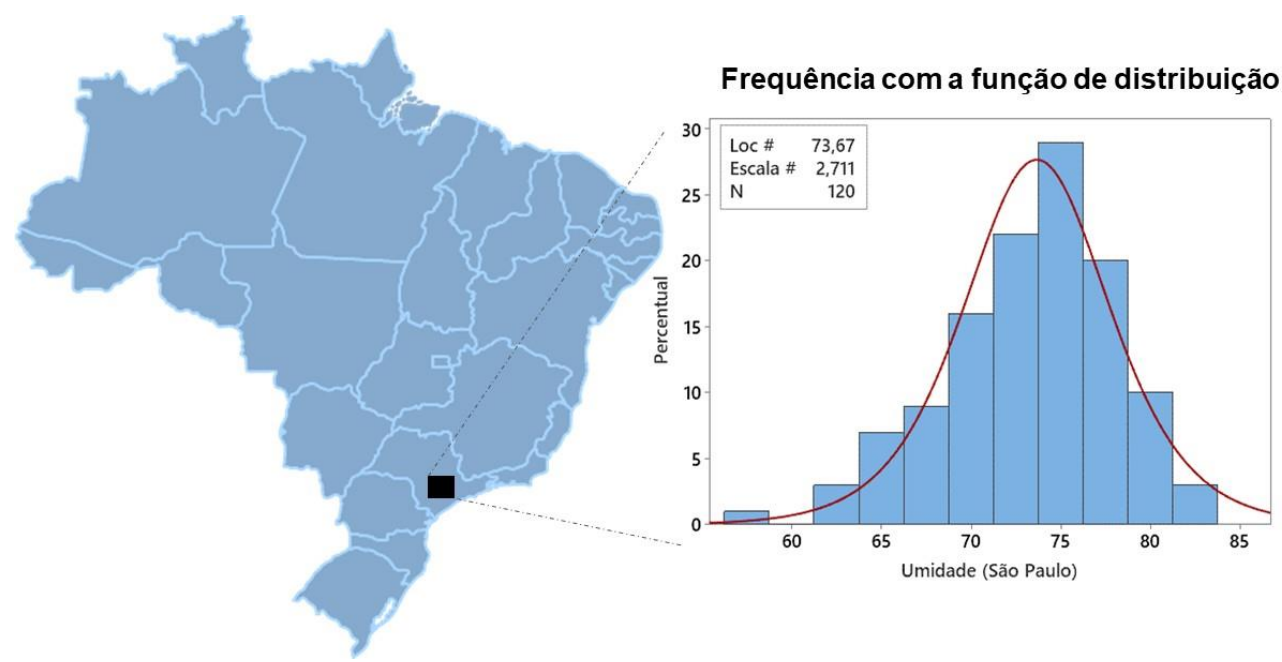

Figura 4: Distribuição dos dados referentes à umidade relativa mensal média (\%).

Para a determinação das funções de distribuição, médias e desvios, realizou-se a coleta de dados do ambiente a ser analisado. Os dados referentes a umidade foram coletados para a capital de São Paulo (SP). Na Figura 4 são apresentadas a frequência de distribuição da umidade média mensal e a função de distribuição determinada por ajustes de curva. Para a construção da curva, utilizou-se os dados referentes ao período 
compreendido entre 02/2010 e 02/2020, sendo extraídos do banco de dados meteorológicos para ensino e pesquisa (BDMEP) do Instituto Nacional de Meteorologia [34]. A Tabela 1 apresenta os resultados obtidos para a média, desvio padrão e a função de distribuição. Para o ajuste de curva, foram testadas as funções de distribuição Normal, Lognormal, Logística, Gama e Weibull.

Para o teor de $\mathrm{CO}_{2}$, foram utilizados dados disponibilizados pelo órgão $\mathrm{CO}_{2}$ Levels [35]. Com os dados foi possível determinar a distribuição das variáveis para o ambiente urbano brasileiro. A média, o desvio e a função de distribuição podem ser visualizados na Tabela 1 .

Para a caracterização da resistência à compressão (Tabela 1), foram utilizados os resultados obtidos no trabalho de HELENE e TERZIAN [36], onde os autores realizaram o levantamento dos diversos níveis de resistência e da variabilidade de concretos produzidos em sete estados brasileiros.

Tabela 1: Parâmetros estatísticos referentes às variáveis aleatórias.

\begin{tabular}{l|c|c|c}
\hline PARÂMETRO & MÉDIA & DESVIO & DISTRIBUIÇÃO \\
\hline Teor de $\mathrm{CO}_{2}(\%)$ & 0,041 & 0,0043 & Normal \\
\hline Umidade média (\%) & 73,39 & 4,80 & Logística \\
\hline Resistência à compressão (MPa) & 27,40 & 3,14 & Normal \\
\hline Cobrimento nominal (mm) & 30,00 & 3,60 & Normal \\
\hline
\end{tabular}

Quanto ao cobrimento nominal das armaduras, adotou-se como média o valor definido como mínimo para um ambiente com classe de agressividade II, conforme prescrito na NBR 6118 [37] para ambientes urbanos (CA II). A definição da função de distribuição e do desvio padrão, apresentados na Tabela 1, foi feita considerando os dados apresentados nos trabalhos de ENRIGHT et al. [38] e LIBERATI et al. [22].

\subsection{Análise probabilística via simulação de Monte Carlo}

$\mathrm{O}$ método de simulação de Monte Carlo é um procedimento numérico, amplamente utilizado em diversas áreas, para a realização de experimentos aleatórios em problemas de confiabilidade. Neste método, amostras de variáveis aleatórias são utilizadas para construir um conjunto de realizações do experimento com o intuito de descrever a falha, os espaços seguros, correlacionados à confiabilidade, e calcular a probabilidade do evento, conforme Equação 4.

$$
P_{f}=\int_{G \leq 0} f_{x}\left(x_{1}, x_{2}, \ldots, x_{n}\right) d x_{1}, d x_{2}, \ldots, d x_{n}
$$

em que $P_{f}$ é a probabilidade de falha, $f_{x}$ é a função conjunta de densidade das variáveis $x_{i}$.

As amostras utilizadas para o cálculo da probabilidade de falha são construídas com base na distribuição estatística atribuída a cada variável aleatória do problema. Como o método está baseado na simulação da função de estado limite, quanto maior for a amostra gerada, mais precisa será a descrição do espaço e mais precisa será a probabilidade de falha ou confiabilidade determinada [39].

Na prática, a avaliação da integral definida na Equação 4 é quase impossível, fazendo-se necessário adotar métodos de cálculo e procedimentos alternativos, desenvolvidos com base no conceito do índice de confiabilidade $\beta$ [39]. O índice de confiabilidade é definido como a distância entre o ponto médio e o ponto de falha alocado sobre a função de estado limite, $G(X)=0$, e está relacionada com a probabilidade de falha por meio da função de distribuição normal padrão acumulada $\phi$, conforme Equação 5.

$$
P_{f}=\phi(-\beta)
$$

De forma geral, o método de simulação de Monte Carlo consiste na construção de uma amostra para as variáveis aleatórias envolvidas no problema a ser simulado, e assim, a probabilidade de falha é calculada utilizando um estimador, baseado na avaliação da função de estado limite, conforme Equação 6. O estimador $I\left(x_{i}\right)$, baseado na função de estado limite, é calculado por meio da Equação 7 , sendo seu valor 0 ou 1 . O valor 0 refere-se ao caso em que não há falha e 1 para quando há falha.

$$
\begin{aligned}
& P_{f}=\int_{G \leq 0} f_{x}\left(x_{i}\right) d x_{i}=\int_{G \leq 0} I\left(x_{i}\right) f_{x}\left(x_{i}\right) d x_{i}=E\left[I\left(x_{i}\right)\right] \\
& I\left(x_{i}\right)= \begin{cases}1, & G(X) \leq 0 \\
0, & G(X)>0\end{cases}
\end{aligned}
$$


Simulando a função de estado limite para um número conveniente de amostras, o valor médio $I\left(x_{i}\right)$ será uma estimativa para a probabilidade de falha. Assim, de acordo com a Equação (8), a probabilidade de falha pode ser facilmente estimada para todo o conjunto de amostras.

$$
\overline{P_{f}}=E\left[I\left(x_{i}\right)\right]=\frac{1}{N} \sum_{i=1}^{N} I\left(x_{i}\right)
$$

em que $N$ é o número de simulações, isto é, de avaliações da equação de estado limite do problema analisado.

Definido o método de estimativa da probabilidade de falha, resta definir a equação de estado limite a ser analisada, $G(X)$. Neste trabalho refere-se à probabilidade de falha por flexão de lajes sujeitas à corrosão desencadeada pela carbonatação, conforme mostra a Equação 9.

$$
G(X)=M_{R}-M_{S}=\left[0,408 b_{w} d^{2} f_{c d} \beta_{x}^{2}+A_{s} \sigma_{S} d\left(1-\beta_{x}\right)\right]-M_{S}
$$

em que $M_{R}$ é o momento resistente $(\mathrm{kN} . \mathrm{m}), M_{S}$ é o momento solicitante $(\mathrm{kN} . \mathrm{m}), d$ é a altura útil (m), $b_{\mathrm{w}}$ é a largura da faixa da laje (utilizado $1,0 \mathrm{~m}), f_{c d}$ é a resistência à compressão de projeto do concreto $\left(\mathrm{kN} / \mathrm{m}^{2}\right), A_{s}$ é a área útil de aço $\left(\mathrm{m}^{2}\right)$ e $\sigma_{s}$ é a tensão de escoamento do aço $\left(\mathrm{kN} / \mathrm{m}^{2}\right)$.

De acordo com a expressão apresentada na Equação 9, tem-se que a falha do evento ocorre quando $G \leq 0$, e não falha quando $G>0$. Assim a falha ocorre quando o momento resistente for inferior ou igual ao momento solicitante. Os demais casos representam a não falha do evento, ou seja, quando o momento resistente é superior ao solicitante.

Por fim, a Figura 5 apresenta um fluxograma que sintetiza os procedimentos e cálculos a serem realizados para estimar a probabilidade de falha, considerando a metodologia proposta baseada na técnica de simulação de Monte Carlo acoplada às formulações analíticas referentes ao processo de degradação das armaduras por corrosão e ao modelo de carbonatação obtido por redes neurais artificias.

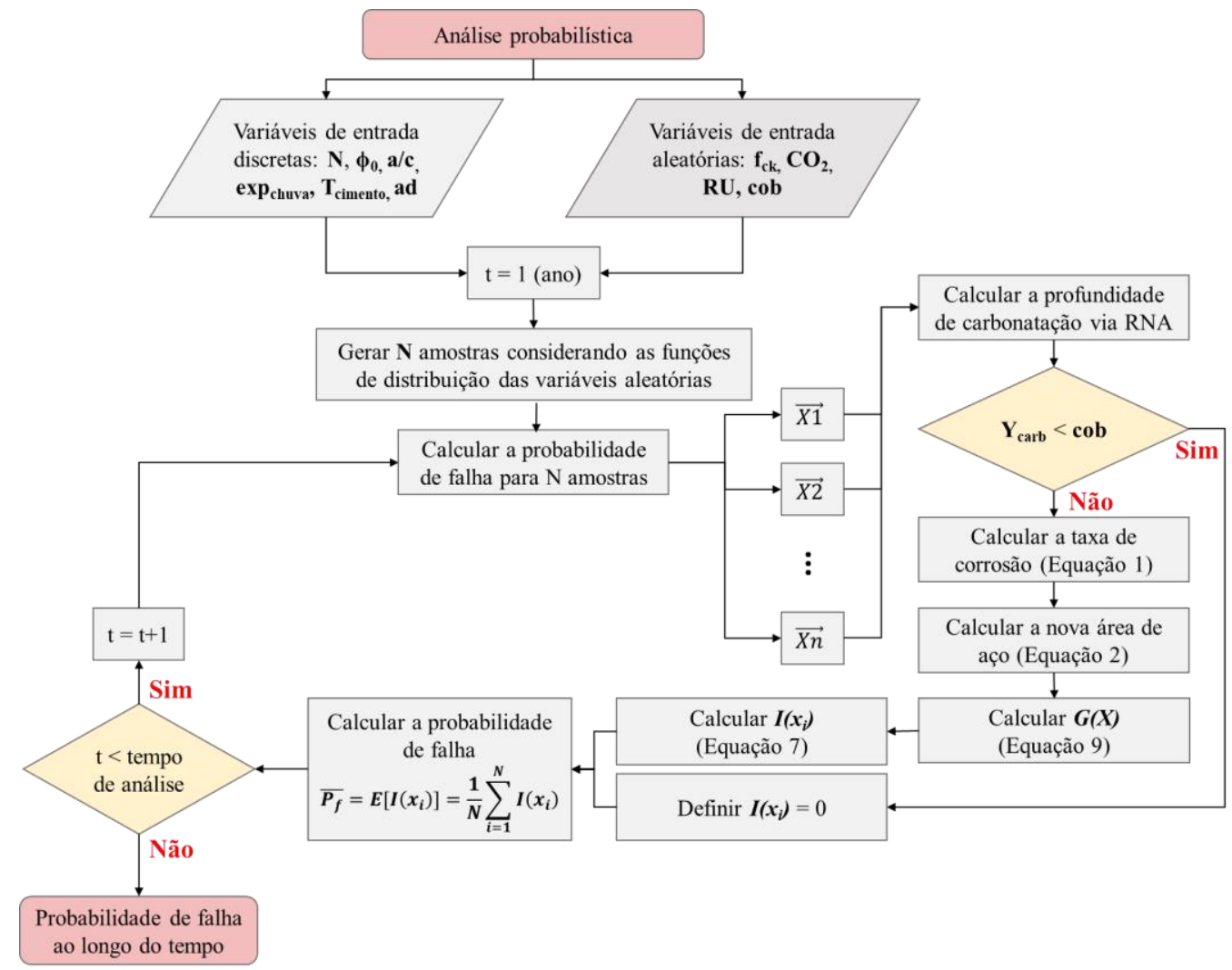

Figura 5: Fluxograma referente à metodologia de análise probabilística da vida útil de estruturas de concreto. 


\section{RESULTADOS}

A fim de analisar a aplicabilidade da metodologia proposta, na sequência são apresentados os resultados obtidos em um estudo de caso, onde é determinada e analisada a falha por flexão de lajes de concreto armado projetadas para a cidade de São Paulo, considerando concretos produzidos com diferentes cimentos e distintas condições de exposição.

\subsection{Estrutura e cenários analisados}

As lajes de concreto armado analisadas neste trabalho, foram dimensionadas de acordo com os procedimentos da NBR 6118 [37], considerando concretos produzidos com os cimentos CP II E, CP II F, CP II Z, CP III, CP IV e CP V. A Figura 6 apresenta um esquema com o carregamento utilizado e a disposição das armaduras.
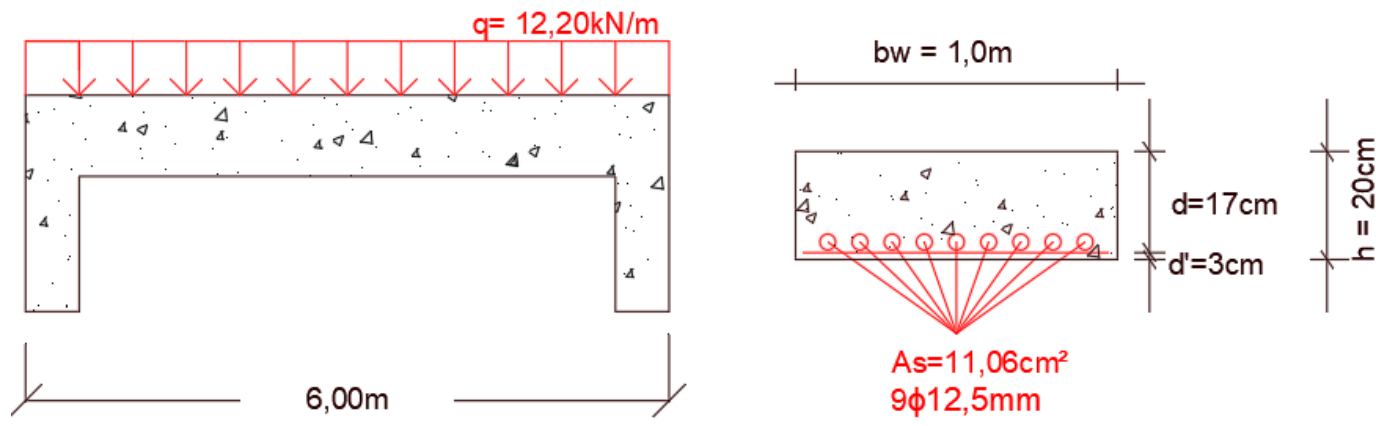

Figura 6: Configuração geométrica da laje de concreto armado.

Para a definição das simulações, as variáveis foram caracterizadas como determinísticas ou aleatórias. As variáveis determinísticas foram divididas em variáveis com valores fixos e não fixos, sendo as variáveis com valores fixos: a altura com $20 \mathrm{~cm}$; largura da seção transversal de $100 \mathrm{~cm}$; barras longitudinais com 12,5 mm de diâmetro; concretos produzidos com relação água/cimento de 0,4 ; resistência à tração do concreto de $0,21 \mathrm{kN} / \mathrm{cm}^{2}$; coeficiente de Poisson do concreto de 0,2; aço CA-50; módulo de elasticidade do aço de 21.000 $\mathrm{kN} / \mathrm{cm}^{2}$; resistência à tração do aço de $50 \mathrm{kN} / \mathrm{cm}^{2}$.

As variáveis determinísticas com valores não fixos foram definidas com o propósito de analisar a sua influência no processo de degradação, sendo dependentes do tipo de cimento (CP II E, CP II F, CP II Z, CP III, CP IV e CP V) e do tipo de exposição (estrutura situada em um ambiente externo protegido ou desprotegida da chuva, ou em ambiente interno protegido da chuva). No total, foram analisadas 18 lajes de concreto armado, considerando os seis tipos de cimento e os três ambientes de exposição.

No que diz respeito às variáveis aleatórias, neste trabalho é analisada a variabilidade presente em quatro parâmetros relacionados à corrosão: (i) o cobrimento das armaduras; (ii) a resistência à compressão do concreto; (iii) a umidade relativa e; (iv) o teor de $\mathrm{CO}_{2}$ no ambiente. Os valores das médias e desvios e, as funções de distribuições foram apresentadas na Tabela 1 .

\subsection{Resultados das simulações}

Inicialmente, na Figura 7 são apresentados os resultados referentes a uma análise determinística do avanço da profundidade de carbonatação do concreto para os seis tipos de cimento Portland (CP II E, CP II F, CP II Z, CP III, CP IV e CP V) e os diferentes ambientes de exposição simulados (ambiente interno protegido da chuva, externo protegido da chuva e externo desprotegido da chuva). Os resultados foram obtidos considerando os valores médios das variáveis aleatórias do problema, conforme Tabela 1, por meio do modelo obtido via redes neurais artificiais [33].

É possível notar, pela Figura 7, que independentemente do tipo de cimento empregado, não há ocorrência de despassivação do aço por carbonatação para estruturas situadas em ambientes externos desprotegido da chuva. No entanto, estruturas situadas em ambientes internos e protegidas da chuva, sofrem despassivação em todos os casos, com exceção da estrutura produzida com cimento $\mathrm{CP} V$.

Acrescenta-se que, para os casos onde as lajes estão em um ambiente interno protegido da chuva, o tempo de despassivação é dado em ordem crescente para os cimentos CP IV, CP III, CP II Z, CP II E e CP II F, apresentando respectivamente, 30, 36, 52, 58 e 61 anos. Analisando os ambientes externos protegidos da chuva, observa-se que apenas as lajes com os cimentos CP III e CP IV têm a despassivação ocorrendo dentro 
do período superior de vida útil de projeto (75 anos). Se analisado o período mínimo de 50 anos, apenas as estruturas situadas em ambientes internos e protegidos da chuva, produzidos com cimentos CP III e CP IV sofreriam a despassivação das suas armaduras.
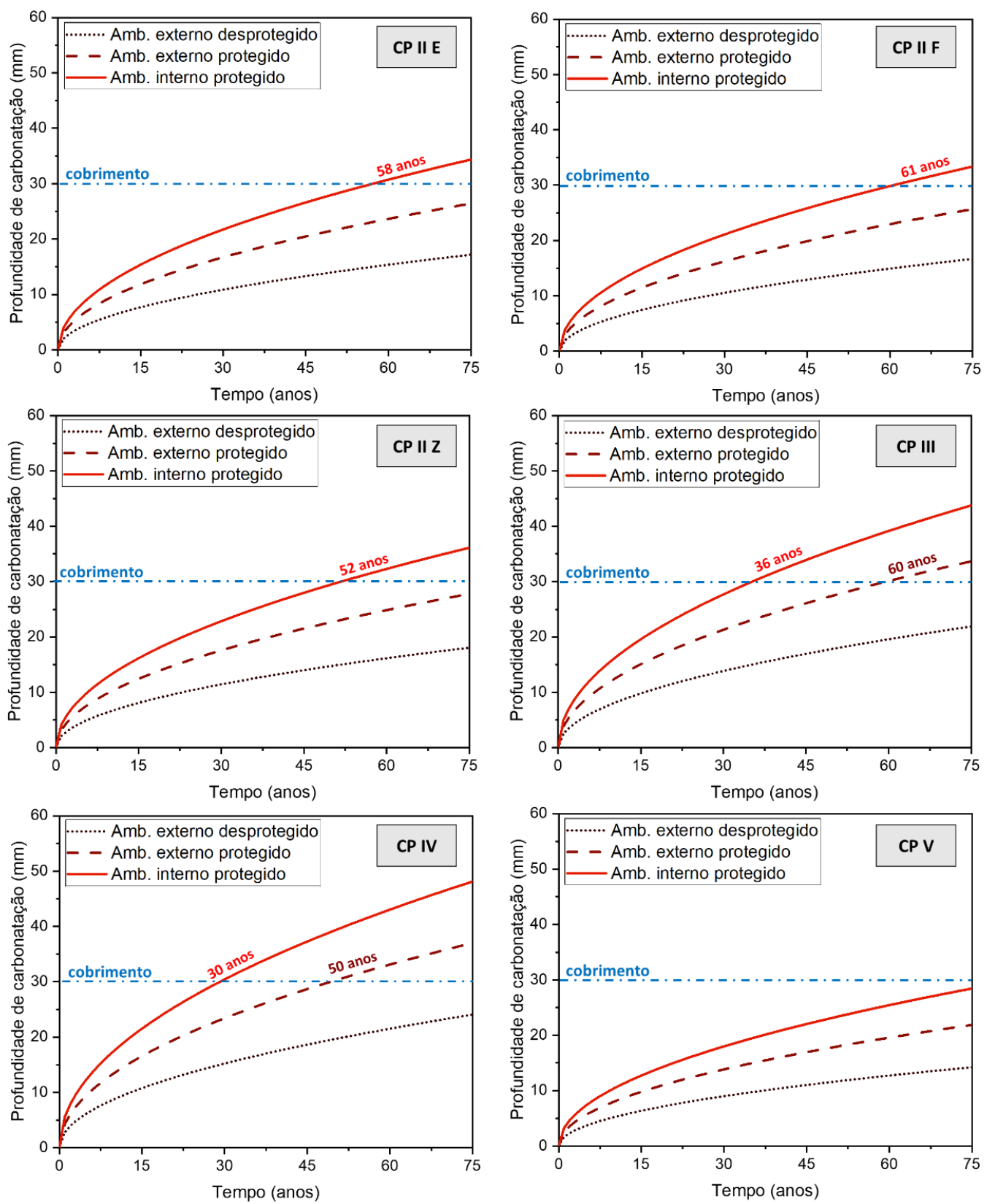

Figura 7: Avanço da profundidade de carbonatação considerando os diferentes cenários de análise.

Dando continuidade à análise determinística, a Figura 8 apresenta a perda de seção resistente de aço e, os momentos resistente e solicitante ao longo do tempo.

Observa-se na Figura 8 que, durante a fase de iniciação da corrosão, a área útil das armaduras não é alterada. A partir do momento em que ocorre a despassivação das armaduras, a perda de seção acontece de forma contínua e não linear, diante da consideração que nenhuma manutenção ou reparo é realizado no elemento estrutural, comportamento coerente com o observado por VU e STEWART [31]. Os autores analisaram por meio de experimentos que a perda de seção de aço ocorre com maior intensidade nos primeiros anos da manifestação patológica, e que, se o processo de degradação não é cessado, a perda de seção útil pode chegar a $50 \%$ em 10 anos.

Considerando as simulações realizadas ao longo de um período de 10 anos de progressão da corrosão 
(período pós-despassivação), verificou-se que a perda de seção de aço varia entre 38 e $48 \%$ e que este valor é dependente do tipo de cimento empregado e do ambiente de exposição. Sendo que as lajes produzidas com cimentos CP III e CP IV teriam as menores vida útil, apresentando ruptura aos 47 e 42 anos, tempo inferior ao inferido pela norma de desempenho NBR 15575-1 [32], como vida útil de projeto de estruturas de concreto.
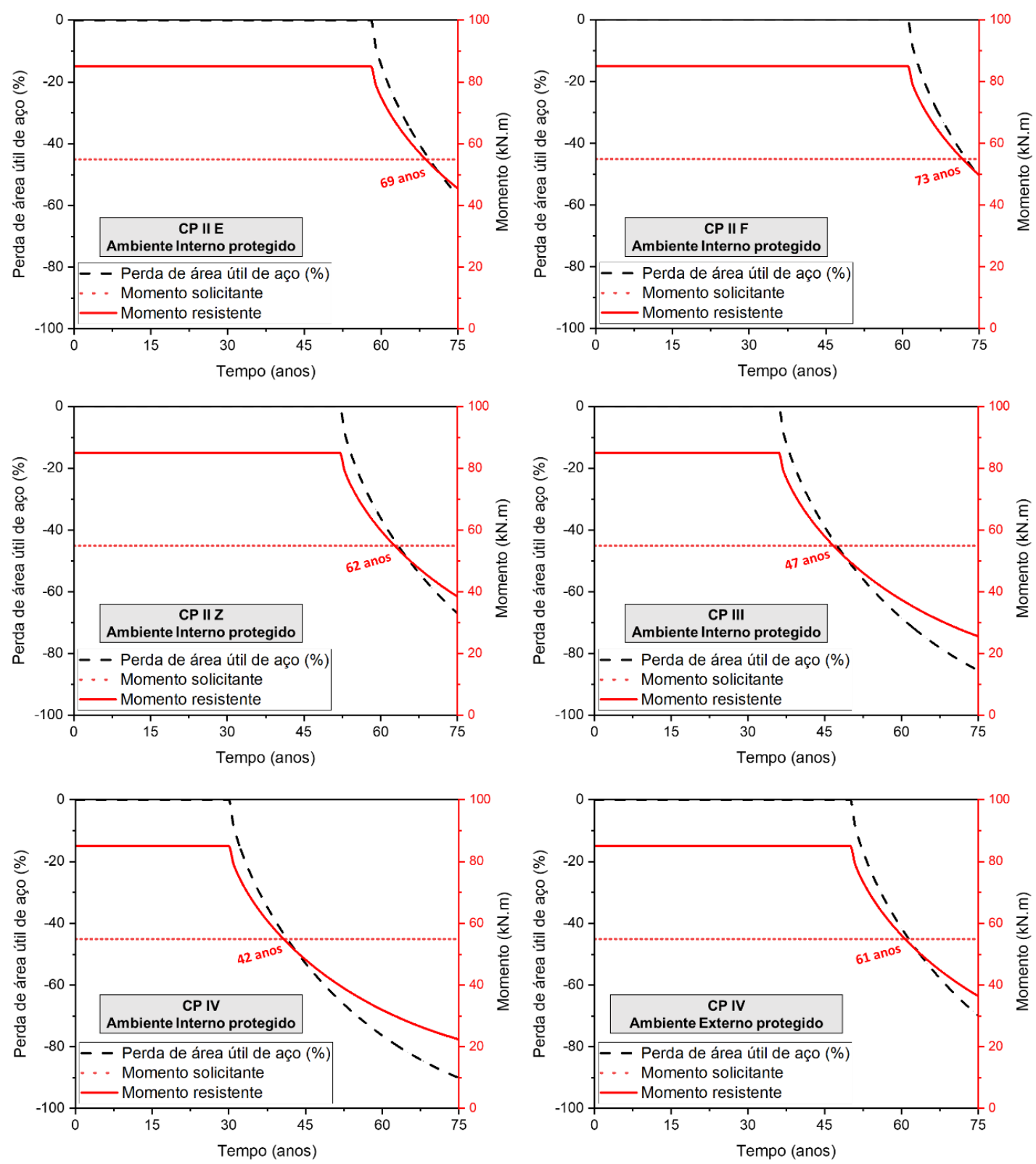

Figura 8: Perda de seção útil de aço e curvas de momento resistente versus momento solicitante ao longo do tempo.

Dando continuidade na análise dos resultados apresentados na Figura 8, nota-se que a falha estrutural das lajes por flexão ocorre para todos os casos onde foi constatada a ocorrência da despassivação do aço (Figura 7). Na análise determinística, observou-se que a falha estrutural só ocorre nas lajes com cimento CP III e CP IV situadas em ambiente interno protegido da chuva, considerando o tempo mínimo de 50 anos. Quando considerado o tempo superior de 75 anos, as lajes produzidas com cimento CP II E, CP II F e CP II Z também teriam sua segurança comprometida, tanto para o ambiente interno protegido da chuva quanto para o externo protegido da chuva.

Após as análises determinísticas, a falha das lajes foi analisada probabilisticamente, sendo necessário a priori, determinar o número de amostras e simulações a serem realizadas, de forma a obter resultados confiáveis. De acordo com LIBERATI et al. [22], para a modelagem de estruturas civis, onde a probabilidade de 
falha está entre $10^{-3}$ e $10^{-6}$, torna-se necessário a realização de $10^{5}$ a $10^{9}$ simulações e análises das funções de estado limite que definem o problema, ou então, realizar um estudo de convergência para cada equação, alternativa adotada neste trabalho. Assim, na Figura 9 são apresentados os resultados de uma análise de convergência, referente à variabilidade do índice de confiabilidade com o número de amostras empregado nas simulações.

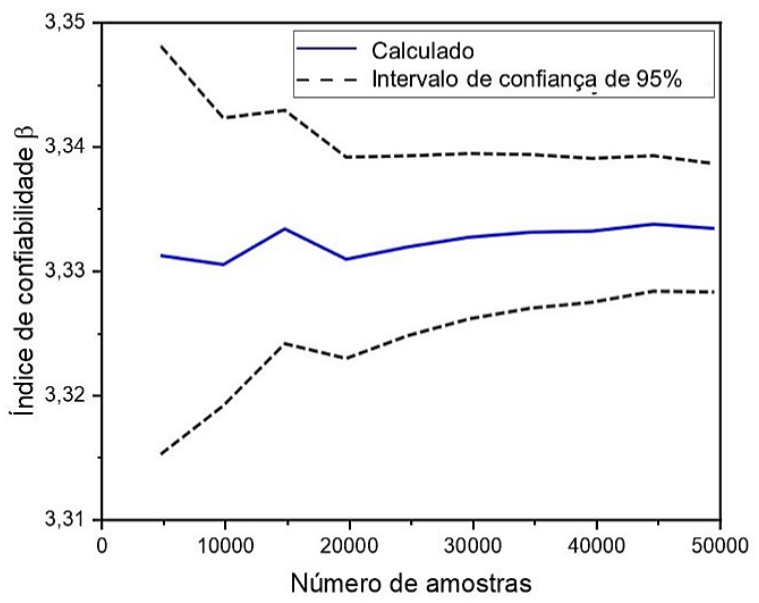

Figura 9: Índice de confiabilidade versus número de amostras para o problema estudado.

Por meio dos resultados apresentados na Figura 9, é possível inferir que para um número de amostras superior a 30.000, o índice de confiabilidade, e por consequência, a probabilidade de falha (Equação 5) tende a se estabilizar. Desta forma, foram empregadas neste trabalho, 30.000 amostras os quais geraram 30.000 simulações para cada uma das lajes.

Determinado o número de amostras, pôde-se realizar as simulações para a estimativa da probabilidade de falha das estruturas. A Figura 10 apresenta as probabilidades de as estruturas atingirem o estado limite último durante o período que compreende a vida útil de projeto.

A partir dos resultados dispostos na Figura 10, verificou-se que a probabilidade de falha é aproximadamente nula para os 15 primeiros anos de vida, independentemente do tipo de cimento e do ambiente de exposição, já que neste período a ocorrência de despassivação é descartada.
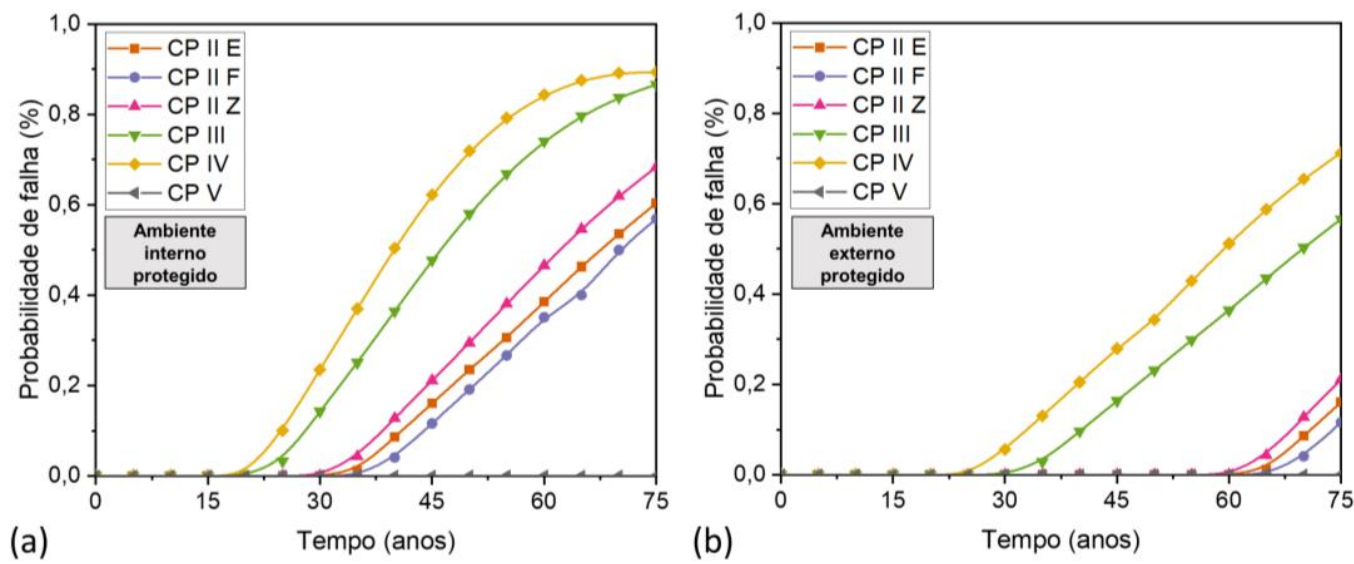

Figura 10: Probabilidade de falha considerando uma laje em um ambiente (a) interno e (b) externo.

Para as estruturas de concreto situadas em ambiente interno e protegidas da chuva, a ocorrência de falha se mostrou elevada, alcançando probabilidades de 50\% (correspondente a um índice de confiabilidade nulo) aos 36-65 anos, dependendo do tipo de cimento empregado. Como esperado, os cimentos CP IV e CP III foram os que geraram as maiores probabilidades de falha, alcançando $50 \%$ aos 36 e 45 anos, respectivamente.

Quando analisadas as lajes em ambiente externo e protegidas da chuva, se nota que a despassivação até os 50 anos, período mínimo de vida útil de projeto descrito na NBR 15575-1 [32], é inferior aos 10\%, 
valor correspondente à um índice de confiabilidade de 1,3, para os cimentos CP V, CP II E, CP II F e CP II Z. Nestes casos, os resultados mostram que as estruturas produzidas atendem aos requisitos mínimos de durabilidade exigidos em norma [32, 37].

Diante das Figuras 10.a e 10.b, observa-se que as estruturas produzidas com cimentos CP III e CP IV apresentam as maiores probabilidades de despassivação por carbonatação e menor durabilidade. JIANG et al. [40] e POSSAN et al. [41] reportam a existência de uma influência negativa de adições no avanço da frente de carbonatação, decorrente da redução da reserva alcalina do concreto quando produzido com elevados teores de escória (de 35 a 70\%) e pozolana (de 15 a 50\%) em suas composições. Ademais os concretos produzidos com cimentos CP III e CP IV apresentaram as maiores probabilidades de falha, sendo $100 \%$ superiores aos concretos produzidos com os demais tipos de cimento, com curvas de probabilidade iniciando na metade do tempo observado para os cimentos CP II E, CP II Z e CP II F. Para o cimento CP V não foram identificadas probabilidades de falhas observáveis para todos os ambientes de exposição estudados, considerando o período de análise de 75 anos.

Infere-se da Figura 10 que, embora os cimentos CP II E e CP II Z tenham adições em sua composição (escória e pozolana, respectivamente), as profundidades de carbonatação são inferiores às obtidas nas vigas produzidas com os cimentos CP III e CP IV, devido ao fato dos aglomerantes CP II E e CP II Z apresentarem teores de adição significativamente menores do que os cimentos CP III e CP IV. Deste modo, observa-se que a composição química do cimento possui influência significativa na velocidade de carbonatação devido à quantidade de compostos alcalinos disponíveis para reagir com $\mathrm{CO}_{2}$, reação química que caracteriza a carbonatação.

KIRCHHEIM [42] e CASTELLOTE et al. [43] relatam que a velocidade de carbonatação está condicionada à quantidade de hidróxido de cálcio disponível na pasta de cimento, tornando a carbonatação função do tipo de cimento empregado na produção do concreto. De acordo com o modelo desenvolvido por POSSAN et al. [41], a velocidade de difusão do $\mathrm{CO}_{2}$ no concreto varia em função do tipo de cimento empregado, apresentando intensidades decrescente nos concretos produzidos com CP IV, CP III, CP II Z, CP II E, CP II F e CP V, resultados coerentes com os obtidos no presente trabalho.

Por fim, confrontando os resultados apresentados nas Figuras 7, 8 e 10, se nota a necessidade da incorporação de análises probabilísticas no estudo de durabilidade e vida útil, já que, se fossem analisadas apenas os valores médios dos parâmetros, não ocorreria falha para a maioria das estruturas (considerando o período mínimo de vida útil), com exceção das lajes inseridas em ambiente interno e produzidas com cimentos CP III e CP IV. Isso não é observado nas análises probabilísticas, onde probabilidades de falha superiores a $20 \%$ são vistas para instantes de tempo inferior aos 50 anos, indicando que, se analisada a variabilidade dos parâmetros executivos e ambientais, as estruturas podem não atender aos requisitos mínimos de durabilidade requisitados em projeto.

\section{CONCLUSÕES}

No presente trabalho foi explorada e proposta a aplicação de uma metodologia alternativa para a análise probabilística da vida útil de estruturas de concreto sujeitas à corrosão por carbonatação. O método foi proposto considerando o acoplamento da técnica de simulação de Monte Carlo acoplada a formulações analíticas de representação da corrosão a um modelo numérico da carbonatação do concreto, baseado em redes neurais artificiais.

De modo geral, os resultados encontrados no estudo de caso, demonstram a eficiência da técnica proposta, apontando a sua aplicabilidade para o estudo de durabilidade e de vida útil de estruturas de concreto armado, considerando a variabilidade dos parâmetros representativos da corrosão.

Com base nos resultados obtidos no estudo de caso, pôde-se elencar as seguintes considerações:

- Um cobrimento adequado garante não só um período maior para a ocorrência da despassivação da armadura, como também para atingir o estado limite último do elemento de concreto;

- Se apenas considerada a perda de seção da armadura como consequência da corrosão, a capacidade mecânica pode ser reduzida pela metade em um período de 10 a 15 anos após o início da corrosão;

- Concretos produzidos com cimentos que conferem maior durabilidade, por exemplo CP V, apresentam maior resistência à carbonatação e, consequentemente, à corrosão, o que leva a um alto índice de confiabilidade;

- Diante das análises, observou-se que das quatro variáveis aleatórias empregadas na modelagem, o cobrimento nominal das armaduras e a resistência à compressão foram as que apresentaram maior relevância e sensibilidade frente à falha da estrutura por flexão; 
- A fim de garantir maior durabilidade aos componentes estruturais de concreto armado, recomendase utilizar cimentos Portland do tipo CP V, CP II F, CP II E e CP II Z para a produção de estruturas inseridas em ambientes com grande concentração de $\mathrm{CO}_{2}$.

Os resultados apontam que a durabilidade de estruturas de concreto armado sujeitas à corrosão desencadeada pela carbonatação, deve ser associada à espessura de cobrimento das armaduras, permeabilidade do concreto, tipo de cimento e o ambiente de exposição.

Ademais, os resultados deste trabalho indicam que abordagens puramente determinísticas não são capazes de avaliar adequadamente a vida útil de estruturas de concreto sujeitas ao processo de corrosão, sendo necessário analisar o problema considerando a aleatoriedade dos diferentes parâmetros. Outrossim a utilização de métodos probabilísticos, em especial a metodologia proposta, indica uma eficiente alternativa para a avaliação da vida útil e durabilidade de estruturas de concreto.

\section{AGRADECIMENTOS}

Os autores agradecem ao Conselho Nacional de Desenvolvimento Científico e Tecnológico (CNPq) pelo fomento fornecido à pesquisa (CNPq 141078/2018 e CNPq 310564/2018-2). Este trabalho também foi financiado pelo Programa de Pesquisa Produtividade da Estácio de Ribeirão Preto e pela Coordenação de Aperfeiçoamento de Pessoal de Nível Superior (CAPES código 001).

\section{BIBLIOGRAFIA}

[1] ASLANI, F., DEHESTANI, M., "Probabilistic impacts of corrosion on structural failure and performance limits of reinforced concrete beams", Construction and Building Materials, v. 265, pp. 120316, 2020. https://doi.org/10.1016/j.conbuildmat.2020.120316

[2] ELLINGWOOD, B.R., FRANGOPOL, D.M., "Introduction to the State-of-the-Art Collection: RiskBased Lifecycle Performance of Structural Systems", https://doi.org/10.1061/(ASCE)ST.1943541X.0001522 . Journal of Structural Engineering, v. 142, pp. 1-15, 2016.

[3] KARI, O.P., PUTTONEN, J., SKANTZ, E., "Reactive transport modelling of long-term carbonation", https://doi.org/10.1016/j.cemconcomp.2014.05.003. Cement and Concrete Composites, v. 52, pp. 42-53, 2014.

[4] FELIX, E.F., Modelagem da Deformação do Concreto Armado Devido à Formação dos Produtos de Corrosão, Dissertação de M.Sc., Escola de Engenharia de São Carlos, Universidade de São Paulo, São Carlos, 2018.

[5] DAL MOLIN, D.C.C., Fissuras em estruturas de concreto armado: análise das manifestações típicas e levantamento de casos ocorridos no Estado do Rio Grande do Sul, Dissertação de M.Sc., Universidade Federal do Rio Grande do Sul, Porto Alegre, 1988.

[6] ANDRADE, C., Manual para diagnóstico de obras deterioradas por corrosão de armaduras, Tradução de Antônio Carmona e Paulo Helene, São Paulo, PINI, 1992.

[7] BAUER, L.A.F., Materiais de construção 2, 5 ed., Rio de Janeiro, Livros Técnicos e Científicos, 2008.

[8] HELENE, P.R.L., Corrosão em Armaduras para Concreto Armado, São Paulo, IPT, PINI, 1986.

[9] BAKKER, R.F.M., Initiation period. Corrosion of steel in concrete, RILEM, pp. 22-54, 1988.

[10] NEVILLE, A.M., Propriedades do concreto, São Paulo, PINI, 1997.

[11] CHANG, C.F., CHEN, J.W., "The experimental investigation of concrete carbonation depth", https://doi.org/10.1016/j.cemconres.2004.07.025 . Cement and Concrete Research, v. 36, pp. 1760 -1767, 2006.

[12] TUUTTI, K., Corrosion of steel in concrete, Stockholm, Swedish Cement and Concrete Research Institute, 1982.

[13] POSSAN, E., Modelagem da carbonatação e previsão de vida útil de estruturas de concreto em meio urbano, Tese de D.Sc., Programa de Pós-Graduação em Engenharia Civil, Universidade Federal do Rio Grande do Sul, Porto Alegre, 2010.

[14] BROOMFIELD, J.P., Corrosion of steel in concrete: understanding, investigation and repair, CRC Press, 2003. 
[15] KÖLIÖ, A., PAKKALA, T.A., HOHTI, H., et al. "The corrosion rate in reinforced concrete facades exposed to outdoor environment", https://doi.org/10.1617/s11527-016-0920-7. Materials and Structures, v. 50, pp. 1-16, 2017.

[16] DU, X., LIU, J., "Meso-scale numerical investigation on cracking of cover concrete induced by corrosion of reinforcing steel", https://doi.org/10.1016/j.engfailanal.2014.01.011. Engineering Failure Analysis, v. 39 pp. 21-33, 2014.

[17] ANDRADE, C.A.C., DIEZ, J.M.A., ALONSO, C.A., "Mathematical Modeling of a Concrete Surface Skin Effect on Diffusion in Chloride Contaminated Media", https://doi.org/10.1016/S1065-7355(97)00002-3 Advanced Cement Based Materials, v. 6, n. 2, pp. 39-44, 1997.

[18] FELIX, E.F., POSSAN, P., "Modeling the carbonation front of concrete structures in the marine environment through ANN". https://doi.org/10.1109/tla.2018.8444398. IEEE Latin American Transactions, v. 16, n. 6, pp. 1772-1779, 2018.

[19] SILVESTRO, L., ANDRADE, J.J.O., DAL MOLIN, D.C.C., "Evaluation of service-life prediction model for reinforced concrete structures in chloride-laden environments", https://doi.org/10.1007/s41024019-0059-3. Journal of Building Pathology and Rehabilitation, v. 4, n. 1, pp. 1-20, 2019.

[20] WU, L., WANG, Y., WANG, Y., et al. "Modelling of two-dimensional chloride diffusion concentrations considering the heterogeneity of concrete materials", https://doi.org/10.1016/j.conbuildmat.2020.118213 . Construction and Building Materials, v. 243, n. 20, pp. s.n, 2020.

[21] TREVISOL, C.A., SILVA, P.R.P., PAULA, M.M.S., et al. "Avaliação de inibidores de corrosão para estruturas de concreto armado", https://doi.org/10.1590/s1517-707620170004.0238. Matéria (Rio de Janeiro), v. 22, n. 4, pp. s.n., 2017.

[22] LIBERATI, E.A.P., LEONEL, E.D., NOGUEIRA, C.G., "Influence of the reinforcement corrosion on the bending moment capacity of reinforced concrete beams: a structural reliability approach", https://doi.org/10.1590/S1983-41952014000300005. Ibracon Structures and Materials Journal, v. 7, n. 3, pp. 379-413, 2014.

[23] RAMEZANIANPOUR, A.A., TARIGHAT, A., MIYAMOTO, A., "Concrete Carbonation Modelling and Monte Carlo Simulation Method for Uncertainty Analysis of Stochastic Front Depth”, 山口大学工学部 研究報告, v. 50, n. 2, pp. 149-152, 2000.

[24] KIRKPATRICK, T.J., WEYERS, R.E., ANDERSON-COOK, et al., "Probabilistic model for the chloride-induced corrosion service life of bridge decks", https://doi.org/10.1016/S0008-8846(02)00905-5. Cement and concrete research, v. 32, n. 12, pp. 1943-1960, 2002.

[25] LI, Q., LI, K., ZHOU, X., et al. "Model-based durability design of concrete structures in Hong KongZhuhai-Macau sea link project”, https://doi.org/10.1016/j.strusafe.2014.11.002. Structural Safety, v. 53, pp. 1-12, 2015.

[26] SAASSOUH, B., LOUNIS, Z. "Probabilistic modeling of chloride-induced corrosion in concrete structures using first-and second-order reliability methods", https://doi.org/10.1016/j.cemconcomp.2012.05.001. Cement and Concrete Composites, v. 34, n. 9, pp. 1082-1093, 2012.

[27] AL-ALAILY, H.S., HASSAN, A.A., HUSSEIN, A.A., "Probabilistic and Statistical Modeling of Chloride-Induced Corrosion for Concrete Containing Metakaolin", https://doi.org/10.1061/(ASCE)MT.19435533.0002062. Journal of Materials in Civil Engineering, v. 29, n. 11, pp. s.n., 2017.

[28] LIZARAZO-MARRIAGA, J., HIGUERA, C., GUZMÁN, I., et al. "Probabilistic modeling to predict fly-ash concrete corrosion initiation", https://doi.org/10.1016/j.jobe.2020.101296. Journal of Building Engineering, v. 30, pp. s.n., 2020.

[29] PELLIZZER, G.P., Sobre a modelagem numérica da difusão de cloretos no concreto: uma abordagem pelo método dos elementos de contorno com aplicação de modelos de confiabilidade e otimização, Tese de D.Sc., Programa de Pós-Graduação em Engenharia de Estruturas, Escola de Engenharia de São Paulo, Universidade de São Paulo, São Carlos, 2019.

[30] STEWART, M.G., ROSOWSKY, D.V., "Structural safety and serviceability of concrete bridges subject to corrosion", https://doi.org/10.1061/(ASCE)1076-0342(1998)4:4(146) . Journal of Infrastructure Systems, v. 4, pp. 146-155, 1998. 
[31] VU, K.A.T., STEWART, M.G., "Structural reliability of concrete bridges including improved chlorideinduced corrosion models", Structural Safety, v. 22, pp. 313-333, 2000. https://doi.org/10.1016/S01674730(00)00018-7

[32] ASSOCIAÇÃO BRASILEIRA DE NORMAS TÉCNICAS., NBR 15575-1: Edificações habitacionais Desempenho - Parte 1:Requisitos gerais, Rio de Janeiro, 2013.

[33] FELIX, E.F., CARRAZEDO, R., POSSAN, E., "Parametric analysis of carbonation process in reinforced concrete structures through Artificial Neural Networks", http://dx.doi.org/10.21041/ra.v7i3.245. Revista ALCONPAT, v. 7, n. 3, pp. 302 - 316, 2017.

[34] Banco de Dados Meteorológicos para Ensino e Pesquisa, INSTITUTO NACIONAL DE METEOROLOGIA, http:/www.inmet.gov.br/ projetos/rede/pesquisa/. Acessado em abril de 2020.

[35] CO2 levels Data Base, 2 DEGREES INSTITUTE, https://www.co2levels.org/\#sources, Acessado em maio de 2020.

[36] HELENE, P. R., TERZIAN, P., Manual de dosagem e controle do concreto, São Paulo, PINI, 1993.

[37] ASSOCIAÇÃO BRASILEIRA DE NORMAS TÉCNICAS., NBR 6118: Projeto de estruturas de concreto - Procedimento, Rio de Janeiro, 2014.

[38] ENRIGHT, M.P., FRANGOPOL, D.M., "Probabilistic analysis of resistance degradation of reinforced concrete bridge beams under corrosion", https://doi.org/10.1016/S0141-0296(97)00190-9. Engineering structures, v. 20, n. 11, pp. 960-971, 1998.

[39] BECK, A.T., Confiabilidade e segurança das estruturas, São Paulo, Elsevier Campus, 2019.

[40] JIANG, L., LIN, B., CAI, Y., "A model for predicting carbonation of high-volume fly ash concrete", https://doi.org/10.1016/S0008-8846(00)00227-1. Cement and Concrete Research, v. 30, pp. 699-702, 2000.

[41] POSSAN, E., ANDRADE, J.J.O., DAL MOLIN, D.C.C., et al. Model to Estimate Concrete Carbonation Depth and Service Life Prediction. In Hygrothermal Behaviour and Building Pathologies, Springer, Cham, pp. 67-97, 2021. https://doi.org/10.1007/978-3-030-50998-9_4

[42] KIRCHHEIM, A.P., Concreto de Cimento Portland Branco Estrutural: avaliação da carbonatação e absorção capilar, Dissertação de M.Sc., Escola de Engenharia Civil, Universidade Federal do Rio Grande do Sul, Porto Alegre, 2003.

[43] CASTELlOTE, M., FERNANDEZ, L., ANDRADE, C., et al. "Chemical changes and phase analysis of OPC pastes carbonated at different $\mathrm{CO}_{2}$ concentrations", https://doi.org/10.1617/s11527-008-9399-1. Materials and Structures, v. 42, n. 4, pp. 515-525, 2009.

ORCID

Emerson Felipe Felix https://orcid.org/0000-0002-8928-9474

Rogério Carrazedo https://orcid.org/0000-0003-2750-034X 